\title{
Tribology and Crystallinity in pivot bearings of Ventricular Assist Devices
}

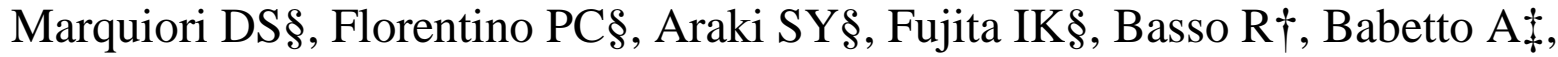 \\ Bonse B $\ddagger$, Moro J§, Leão TF§*, Andrade AJP*, Bock EGP§* \\ $\S$ Federal Institute of Sao Paulo IFSP, Sao Paulo, Brazil. $\dagger$ Federal University for Latin \\ American Integration UNILA, Foz do Iguaçu, Brazil. $\ddagger$ FEI University Center, Sao Bernardo do \\ Campo, Brazil.*Institute Dante Pazzanese of Cardiology, Sao Paulo, Brazil.
}

\begin{abstract}
Ventricular Assist Devices are blood pumps used in patients with Congestive Heart Failure who are waiting for a heart transplant. They aim to assist the ventricle to pump out blood in physiological circulation by increasing aortic pressure and decreasing intraventricular pressure. The IFSP Laboratory of Bioengineering and Biomaterials (BIOENG) has been developing an Implantable Centrifugal Blood Pump called CARoL for mechanical circulatory support. The objective of this dissertation is to evaluate the changes in the crystallinity of the polymeric Pivot Bearings supporting the impeller of this pump when subjected to friction generated by rotation of zirconia oxide ceramic shafts. The adopted methodology consisted of submitting new and used samples of: a) bearings set made of polyamide 6; and b) the set made of poly-ether-ether-ketone. Those new and used samples were characterized by X-ray diffraction tests and Infrared Spectroscopy. The diffractograms and spectra obtained were compared to evaluate the bearing crystallinity, for both polymers before and after friction. The tests carried out showed diffractograms and similar spectra for the new and used samples, thus, there are indications that the friction generated by the rotation of the shafts did not change the crystallinity of the polymeric bearings supporting the pump rotor.
\end{abstract}

Keywords. VAD, X-Ray Diffraction, Infrared Spectroscopy, Crystallinity, Polymeric Bearings, CARoL Pump.

Introduction. Ventricular Assist Devices (VAD) are blood pumps used in patients with Congestive Heart Failure who are waiting in line for a heart transplant. They aim to assist the ventricle to carry out blood circulation by increasing aortic pressure and decreasing intraventricular pressure, which can be applied to the left, right or biventricular assistance [1-5]. It demonstrated through tests carried out in Institute Dante Pazzanese of Cardiology in situations of heart failure resulted in increase of blood flow and reduction in heart rate [6-8].

In 2006, INTERMACS (Interagency Registry for Mechanically Assisted Circulatory Support) was created in the United States, as inter-agency registry with the objective of generating statistical data on mechanical circulatory support. One of the points monitored is the patient's 
survival rate, according to the intended use at the time of implantation (bridge for transplantation, bridge for decision and destination therapy). An important concept is the KaplanMeier curve for continuous flow left ventricular assist devices [9-11].

The IFSP Laboratory of Bioengineering and Biomaterials has been developing an Implantable Centrifugal Blood Pump called CARoL (Cardiac Assistant Recovery of Life) for mechanical circulatory support, illustrated in Figure 1.

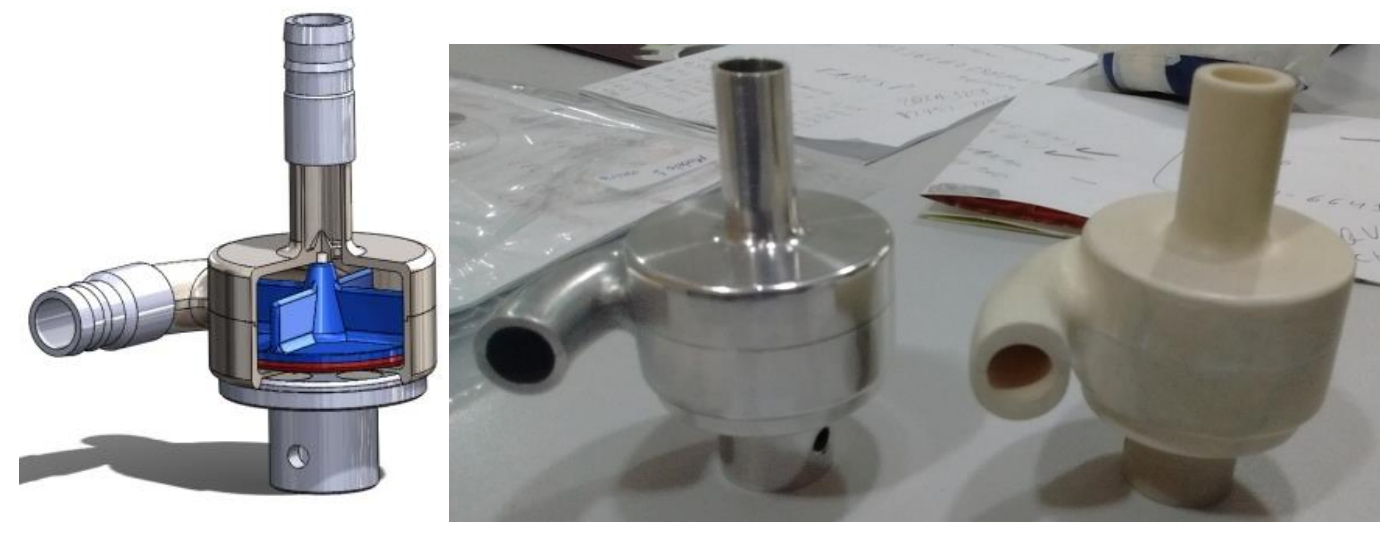

Figure 1. Drawing 3D (left) and Prototypes (right) of the CARoL Pump.

Materials and Methods. The general objective of this work is to evaluate changes in crystallinity of the polymeric pivot bearings of the CARoL pump when submitted to the friction generated by the rotation of the ceramic shafts under application conditions.

The VAD application in patients with Congestive Heart Failure can have a variable duration according to its destination, it can remain implanted for several years in Kaplan-Meier Curve [9].

The design of VADs must be concerned with their durability, in order to minimize the probability of failures and, consequently, the number of interventions due to equipment failure.

The change in crystallinity of polymers generates changes in some properties like Young modulus and hardness.

Thus, a change in pivot bearings crystallinity after use is an indicator of degradation by friction, which can lead to failures in VADs during its lifecycle, Fig. 2. Therefore, the study is justified insofar as it allows identifying the existence or not of alterations in crystallinity. 


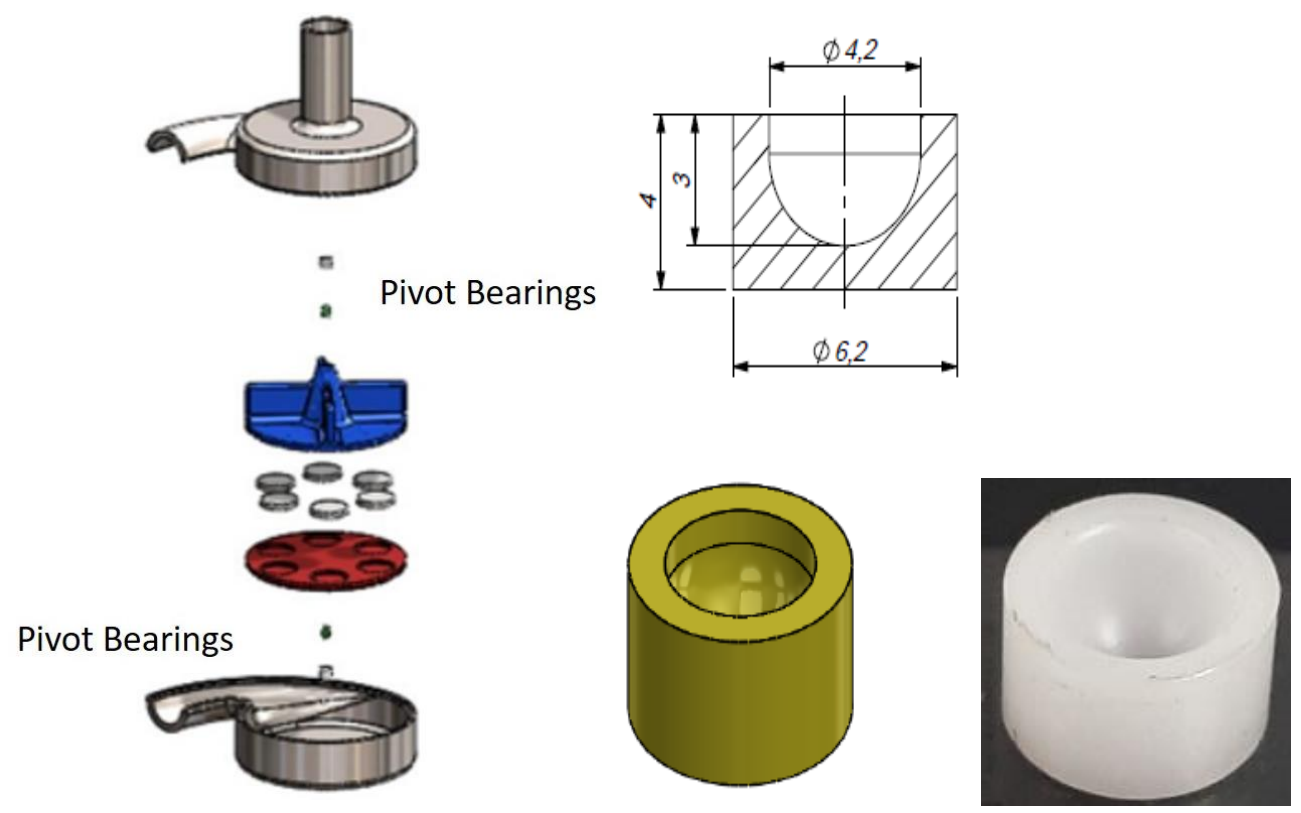

Figure 2. Three-dimensional drawing of CARoL pump showing the position of the pivot bearings (upper and lower) and the machined bearing.

Tests were carried out with zirconia ceramic shafts and PEAUMM, polyamide 6 (PA6) and polyether-ether-ketone (PEEK) bearings. Since VADs are designed for use in long-term ventricular assistance, a system of durable pivot bearings is required, Fig.2. The pivot bearings used remain in contact with the pump shafts only at speeds below 1,100 rpm (lower bearing) and above 1,500 $\mathrm{rpm}$ (upper bearing). In the range of $1,200 \mathrm{rpm}$ to $1,400 \mathrm{rpm}$, hydrodynamic levitation of the rotor occurs, with no friction between the components during normal operation, thus reducing the possibility of wear and reducing the life of the pump [2,6,11].

This work was designed to assess whether there are changes in the crystallinity of the polymeric bearings of the CARoL Pump, two pairs of bearings machined in PA6 and two pairs machined in PEEK were used, each pair consisting of an upper bearing and a lower bearing. The contact area between bearings and ceramic shafts consists of a concave surface in order to guarantee the correct positioning of the shafts and the proper movement of the pump rotor, Fig. 2.

In order to generate results that would allow the comparison of crystallinity after the bearing was used, a new pair and a used pair of bearings from each of the materials were selected to perform the measurements. The used PA6 bearings that were selected for analysis were submitted to 10 hours of tests friction workbench [7]. The fluid applied to the bench to perform the test was pure water [12,13] and after verification with a microscope (up to 200x magnification) and profilometer, no wear was found, only changes in the bearing surfaces $[8,14]$.

PEEK bearings, on the other hand, were submitted to a 6-hour test performed directly on the pump and using $750 \mathrm{ml}$ of human blood as a fluid, which aimed to analyze the hemolysis in the 
CAROL pump. No previous tests were performed to evaluate the wear on these bearings. The tests performed on bench are equivalent to those performed on pumps, since the friction workbench developed has high precision in adjusting the contact force between the bearing and shaft and the motor speed and features a pumping system that simulates the passage of fluid between the bearing and the rotor shaft [15-21].

The new and used samples of the lower bearing of PA6 and of the lower bearing of PEEK were submitted to the X-ray diffraction test (XRD Empyrean Panalytical, Almelo, Netherlands) Fig. 3.

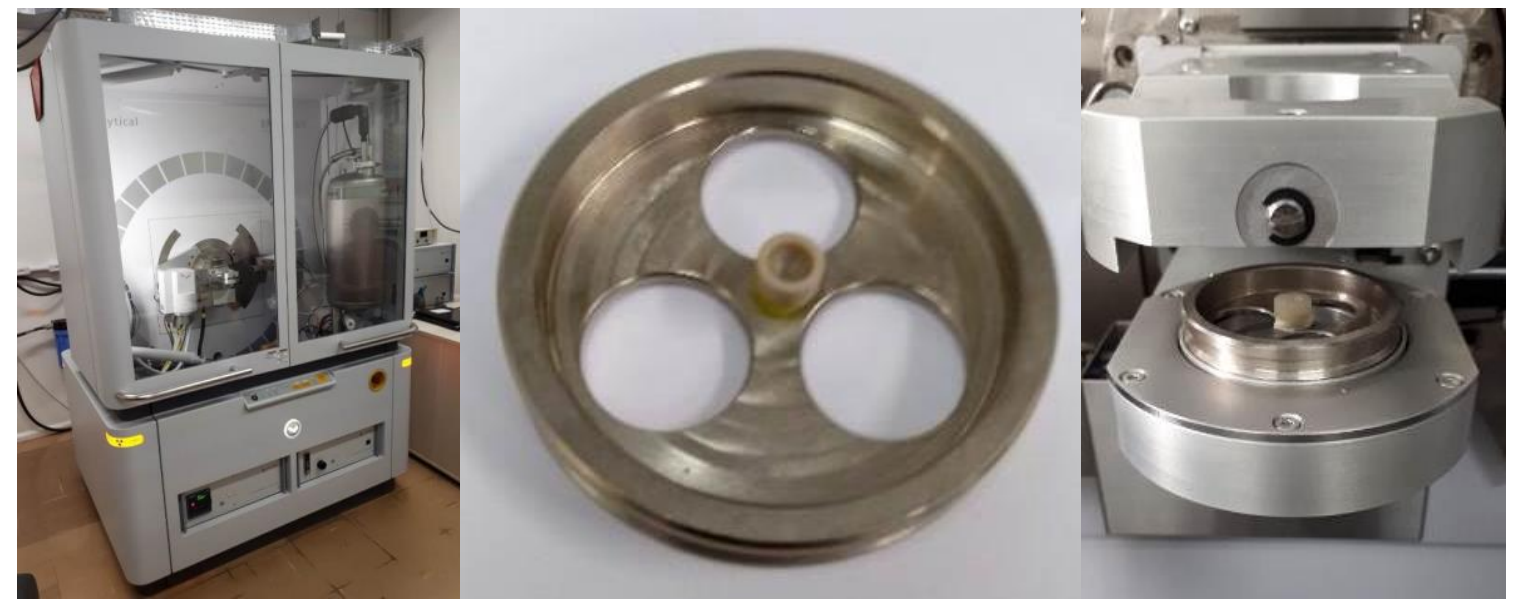

Figure 3. X-ray Diffractometer, sample holder and sample positioning.

The upper bearings were not tested because the shape and dimensions of the samples did not allow the test to be carried out - the ideal is to subject the contact surface between the bearing and the shaft to the test and, in the case of the upper bearing, this does not it is possible without destroying the sample. The specific sample holder was selected for solid sample analysis and each sample was attached to the diffractometer sample holder. Then, the prepared set was positioned on the equipment support for testing, Fig. 3.

The diffractograms obtained were compared with the aid of the High Score Plus software to assess the bearings crystallinity and their changes, for both polymers before and after friction by comparing the position of the diffraction peaks between the diffractograms. Thus, even superficial changes can be perceived in the signature of the materials, depending on the degree of change in the crystallinity of the material, if there is any relevant displacement of the peaks. Changes in the diffraction intensity are not relevant for the evaluation of crystallinity, since it is measured in relative units [22-26].

After X-ray diffraction tests, the upper and lower bearings were subjected to infrared spectroscopy (Nicolet 6700 FT-IR, Thermo Scientific). A first reading was performed on the equipment without any sample, in order to capture the air spectrum and subtract its peaks from 
the sample spectra - to minimize the impact of changes in the air, the laboratory has controlled temperature, humidity and air exchange, in however, the air has high infrared absorption due to its composition (since it contains water and other impurities) and its spectrum must be removed to allow the correct reading of the sample spectrum. Each sample was cleaned with isopropyl alcohol bath, dried on absorbent paper and positioned on the equipment.

Once the sample is positioned, measurement begins, which consists of generating the infrared radiation beam, which falls on the sample so that the equipment measures the radiation absorption by the tested material, generating the characteristic spectrum of that sample. The position of the peaks in terms of wave number is what characterizes a specific band, and consequently, a specific material - the intensity of the peaks has low relevance in the characterization. The spectra obtained were compared in two ways from the point of view of peak positions to assess possible changes between samples of the same material: upper bearing with lower of the same pair, and similar bearings before and after friction [27].

Results. The results obtained by X-ray diffraction (lower bearings) and infrared spectroscopy (upper and lower bearings) tests performed on the new and used samples of the set manufactured in PA6 and the set manufactured in PEEK are presented below.

The PA6 polymer presents diffractograms with two peaks, which are in approximate positions of $20^{\circ}$ and $23^{\circ}$. Therefore, it is the expected behavior for the diffractograms of the tested samples if they do not present differences in crystallinity.

The results may show some variation in intensity, given that it is measured in relative units. Changes in the position of the peaks ( $\mathrm{x}$-axis), and consequently in crystallographic planes, may indicate changes in crystallinity in samples.

Two PA6 samples were submitted to X-Ray Diffraction test according to the methodology described before, the new upper bearing and the used upper bearing, which generated the diffractograms that can be seen in Fig 4.

For both PA6 bearings, the diffractograms show peak intensity at approximately $20^{\circ}$ and $23.4^{\circ}$ positions. There is a variation of less than $0.1^{\circ}$ between the new bearing and the used bearing, which can be disregarded. The $0.4^{\circ}$ variation observed between the reference curve and the spectra does not indicate a variation in the crystallinity of the material. There is a variation in intensity between samples, which can be justified by the concave geometry of the analyzed surfaces, Fig. 4. 


\section{PA6 Pivot Bearings}

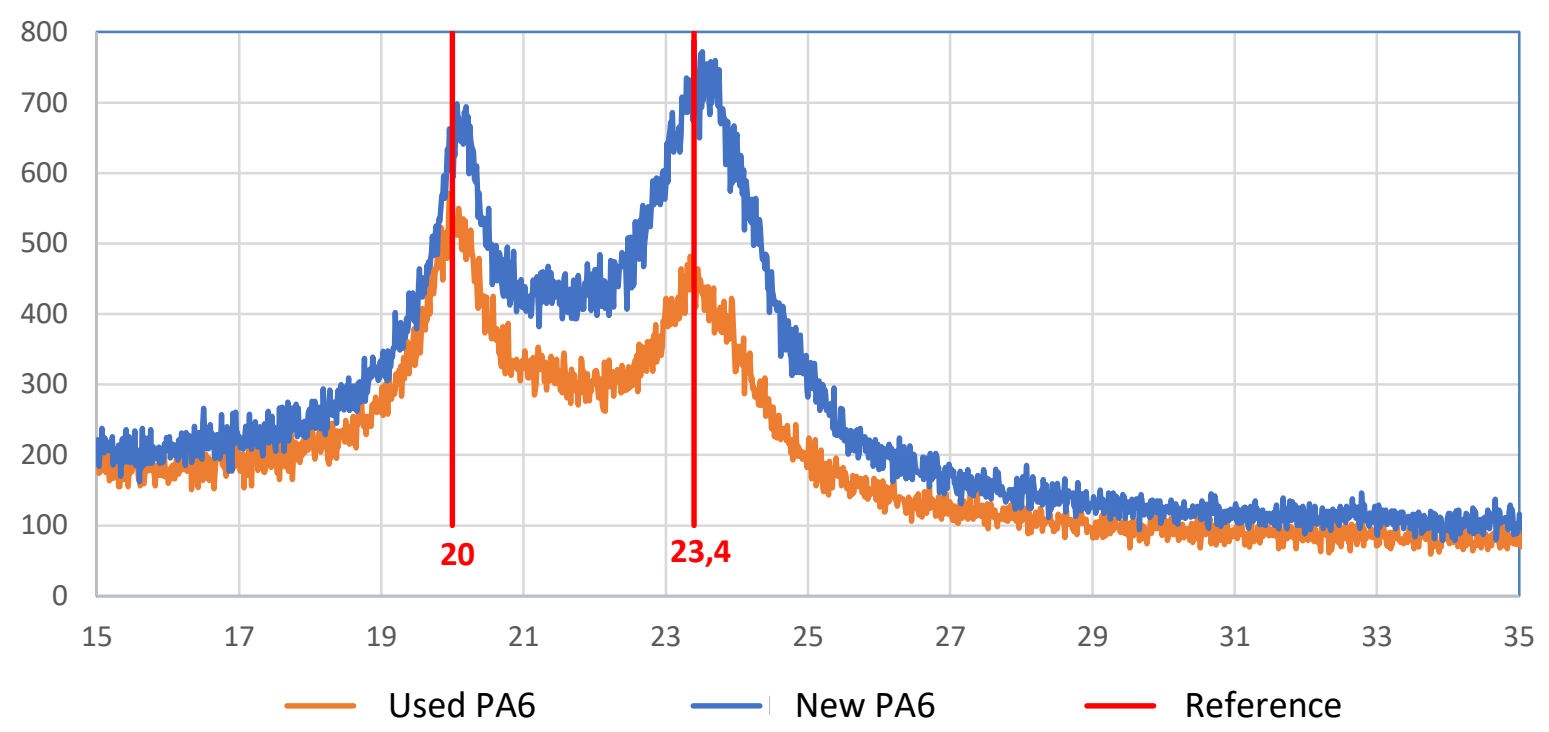

Figure 4. X-ray Diffractometer comparing, used and new PA6 pivot bearings with reference in degrees.

The PEEK reference shows peaks of diffraction intensity visible when $2 \Theta$ is approximately $19^{\circ}$, $21^{\circ}, 23^{\circ}, 29^{\circ}, 33^{\circ}$ and $39^{\circ}$, the first 4 peaks being associated with specific crystallographic planes. As in the PA6 polymer, changes in the diffraction points (x-axis), and consequently in the crystallographic planes, may indicate changes in crystallinity in the samples manufactured in PEEK.

Two PEEK samples were submitted to the X-ray diffraction test according to the methodology described before, the new upper bearing and the used upper bearing, which generated the diffractograms. The PEEK-machined bearings have diffractograms that show four intensity peaks at the $18.7^{\circ}, 20.7^{\circ}, 22.6^{\circ}$ and $28.7^{\circ}$ positions, Fig. 5 .

The peaks in $2 \theta$ equal to $33^{\circ}$ and $39^{\circ}$ were not observed in any of the curves obtained - they are peaks of lesser intensity in relation to the others and may have been reduced to the point of being imperceptible due to variations in diffraction intensity motivated by samples geometry, Fig. 5 .

For PEEK, no diffraction intensity variation was observed between the new sample and the used sample. 


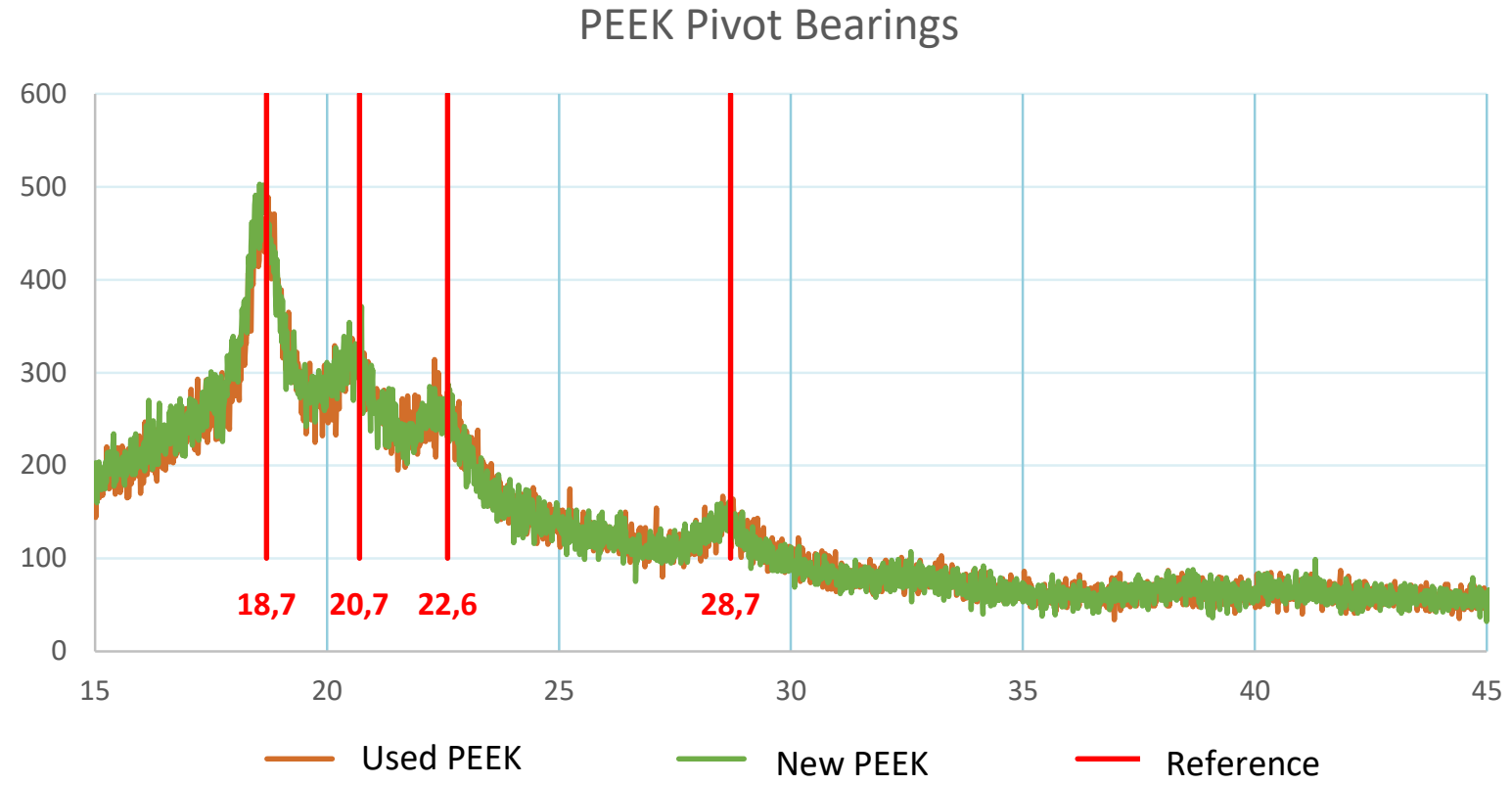

Figure 5. X-ray Diffractometer comparing, used and new PEEK pivot bearings with reference in degrees.

The new and used samples of PA6 submitted to infrared spectroscopy generated the following spectra. In the case of new samples, the comparison between upper and lower bearings shows that most of the absorbance peaks are repeated, with a variation in intensity in some of them and a peak close to $2,960 \mathrm{~cm}^{-1}$ with relevant absorbance (> $0.01 \mathrm{ua}$, according to the reference curves) that appears on the new bearing and does not appear on the used bearings.

Referring to the samples used, when comparing upper and lower bearings, it is noted that the peaks occur in the same positions with variation in absorbance. With these results, a comparative analysis of the new and used samples for each bearing was carried out. All comparisons show some level of difference in absorbance at the peaks. The wave numbers indicated in yellow in Fig. 6. represent the positions of main peaks observed in the reference spectrum, while the one indicated in gray also appears in the reference, but without highlighting depending on its intensity.

The Fig 6. indicates that all the peaks present in the samples appear in the reference curve, with only one of them $\left(830 \mathrm{~cm}^{-1}\right)$ being considered irrelevant in the reference curve, but has an absorbance greater than 0.01 water in the new bearing spectrum. 


\section{Lower PA6 Pivot Bearings}

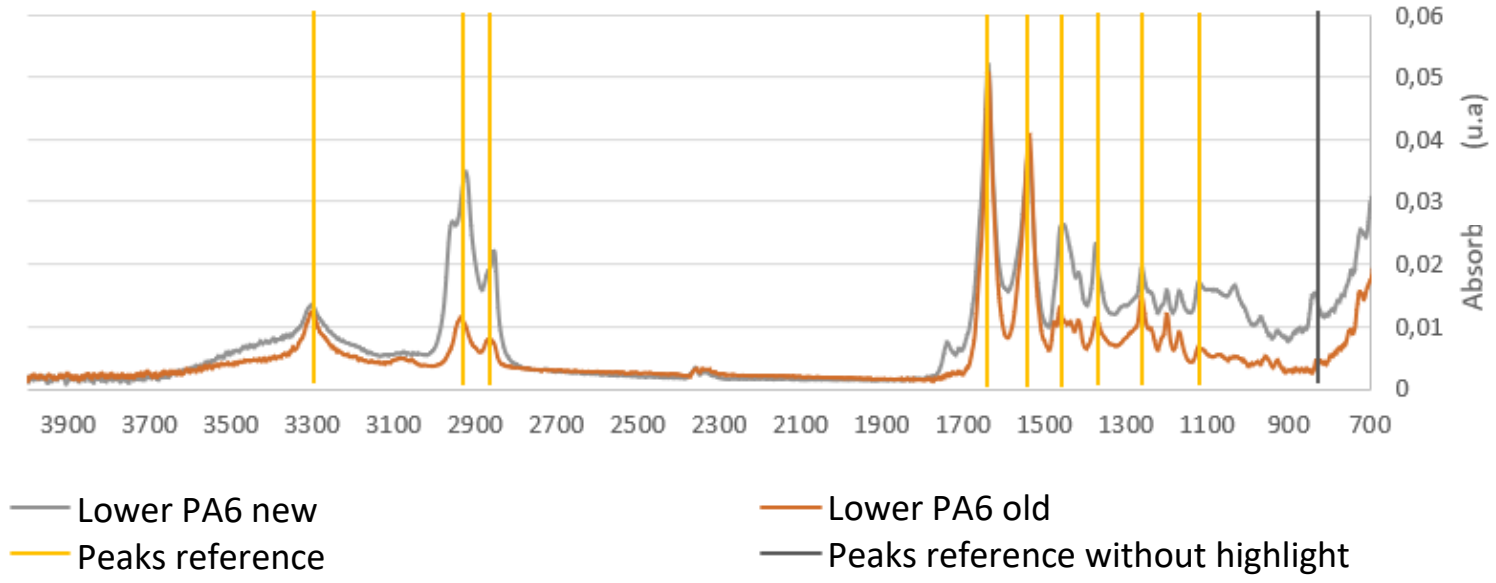

Figure 6. Comparison of the infrared spectra of the lower bearings of PA6.

On the other hand, the comparison of upper bearings shows only yellow lines, that is, the peaks coincide with the reference spectrum.

Upper PA6 Pivot Bearings

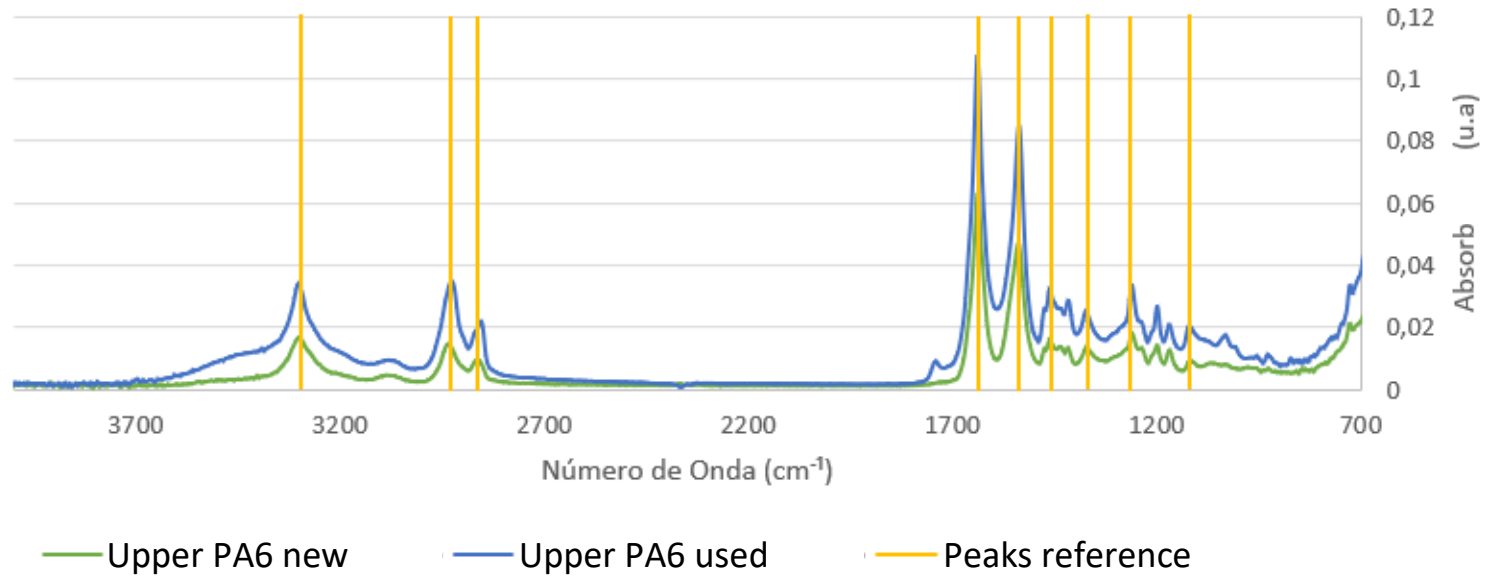

Figure 7. Comparison of the infrared spectra of the upper bearings of PA6.

The new and used PEEK samples submitted to the infrared spectroscopy test generated the following spectra. For new samples, the comparison between upper and lower bearings shows the absorbance peaks at the same positions in the spectrum, with some variations in intensity. For the samples used, the comparison between spectra of the upper and lower bearings shows the absorbance peaks at the same positions and intensities. 
TAS Journal, vol. 4, n. 1, p. 52-62

ISSN 2595-1521

MARCH 2020

drymarquiori@hotmail.com

The wave numbers indicated in yellow represent the main peak positions highlighted by reference spectrum, while those indicated in gray also appear in the reference, but not highlighted due to their intensity. Despite variations in intensity, the absorbance peaks occur in the same positions both in the reference spectrum and in the analyzed samples. The same comparison was made for the PEEK upper bearing samples which shows a lower intensity variation than the lower bearings, with high adherence between the reference spectrum and the samples. In both comparisons, it is possible to notice that the behaviors of the curves are maintained, observing only an increase in the absorbance in used bearings in relation to the new bearings.

Conclusions. In order to evaluate possible changes in the crystallinity of CAROL Pump polymeric bearings when subjected to the friction generated by ceramic axes rotation, X-Ray Diffraction and Infrared Spectroscopy tests were performed on samples of bearings manufactured in PEEK and PA6.

The PA6 bearings generated diffractograms and spectra with peak intensity / absorbance in the same positions for the new and used samples, consistent with the reference curve, showing a small variation in the diffraction intensity, which can be explained by the concave geometry of the analyzed surface and, in the case of spectra, a change in the absorbance levels that do not disfigure the characteristic bands of the material.

Changes due to friction in the materials used for making the bearings were expected. Thus, although in recent work it is possible to measure wear, PEEK samples show diffractograms with peak intensity in the same positions, and diffraction intensities at the same level for the new and used bearings, coinciding with the reference curve. In addition, the spectra show the same absorbance peaks for new and used bearings, also aligned with the reference curve.

Additionally, comparisons were made between upper and lower bearings of the same set, both for PEEK and PA6, using the infrared spectroscopy technique. These comparisons showed slightly different spectra for the PA6 samples, a behavior that remains in both new and used pairs, indicating that the manufacturing process for obtaining the different geometries influences the structure and crystallinity of the material. In the case of PEEK, the pairs have spectra with peaks in the same positions, so there is no evidence of changes in crystallinity between the upper and lower bearings of the same pair.

From the results presented, it is possible to conclude that the friction generated by the rotation of the shaft in the test period of each samples did not change the crystallinity of polymeric bearings supporting the pump impeller in analyzed bearings, in both materials. Therefore, from the point of view of crystallinity, given that both PEEK and PA6 showed the same performance, it is recommended to use PA6, which is a more accessible and cheaper material. 
TAS Journal, vol. 4, n. 1, p. 52-62

ISSN 2595-1521

MARCH 2020

drymarquiori@hotmail.com

Acknowledgments. This work was only possible with financial funding of CAPES, CNPq, and FAPESP. Authors acknowledge Brazilian Funding Agencies CAPES PGPTA 88887.123938/2014-00, CNPq 310085/2015-2, FAPESP PIPE/PITCHGOV 2017/25233-9.

Disclosure. The authors report no conflicts of interest in this work.

\section{References}

(1) Uebelhart B, Silva B, Fonseca J, Bock E, Leme J, Silva C, Leão T, Andrade A. Study of a Centrifugal Blood Pump in a Mock Loop System. Artif Organs 2013;37(11):946-9.

(2) Bock E, Leão T, Fonseca J, Andrade A. Left Ventricle Failure and Blood Flow Estimation for Centrifugal Blood Pumps. J Mech Eng Aut 2016;6:162-6.

(3) Thunberg CA, Gaitan BD, Arabia FA, Cole DJ, Grigore AM. Ventricular Assist Devices Today and Tomorrow. J Cardiotho Vasc Anesth 2010;24(4):656-680.

(4) de Bakey ME. The odyssey of the artificial heart. Artif Organs 2000;24:405-411.

(5) de Souza RL, Chabu IE, Drigo E, de Andrade AJP, Leao TF, Bock EGP. A strategy for designing of customized electromechanical actuators of blood pumps. Artif Organs, 2019.

(6) Bock E, Ribeiro A, Silva M, Antunes P, Fonseca J, Legendre D, Leme J, Arruda C, Biscegli J, Nicolosi D, Andrade A. New Centrifugal Blood Pump With Dual Impeller and Double Pivot Bearing System: Wear Evaluation in Bearing System, Performance Tests, and Preliminary Hemolysis Tests. Artif Organs 2008;32(4):329-333.

(7) Araki SY, Florentino PC, Saito MA, Bock EGP, Arruda ACF, Rocha, J. A., Stoeterau, R. L. Ensaios em materiais cerâmicos e poliméricos de alta performance em mancais nos Dispositivos de Assistência Ventricular (DAV). TAS Journal 2017;1(1):3-7.

(8) Saito M, Araki S, Stoeterau R, Bock E. Estudo de análise superficial em materiais poliméricos para uso em biomedicina. TAS Journal 2017;1(2):60-7.

(9) Kormos RL, Cowger J, Pagani FD, Teuteberg JJ, Goldstein DJ, Jacobs JP, Higgins RS, Stevenson LW, Stehlik J, Atluri P, Grady KL, Kirklin JK. The Society of Thoracic Surgeons Intermacs Database Annual Report: Evolving Indications, Outcomes, and Scientific Partnerships. Ann Thor Surg 2019;107(2):341-353.

(10) Akkanti B, Castriotta RJ, Sayana P, Nunez E, Rajapreyar I, Kumar S, Majid R. Ventricular assist devices and sleep-disordered breathing. Sleep Med Rev 2017;35:51-61.

(11) Bock E. Review of Introductory Tests to in Vivo Evaluation, Prototypes Assembling and Anatomical Position Studies after Five Years. Int J Adv Robot Automn, 2016;1(3):1-3.

(12) Santos BJ, Tabacow RP, Barboza M, Leão TF, Bock E. Cyber Security in Health: Standard Protocols for IoT and Supervisory Control Systems. Cyber Security of Industrial Control Systems in the Future Internet Environment. IGI Global, 2020;313-329.

(13) Braunwald E. Braunwald's Heart Disease: A Textbook of Cardiovascular Medicine. Boston, Elsevier, 2012. 
(14) Kosaka R, Maruyama O, Nishida M, Yada T, Saito S, Hirai S, Yamane T. Improvement of Hemocompatibility in Centrifugal Blood Pump With Hydrodynamic Bearings and Semi-open Impeller: In Vitro Evaluation. Art Organs, 2009;33(10):798-804.

(15) Santos FSF, Ferreira VP, Sá MD, Fook MV. Modificação da superfície do poli (éter-étercetona). Rev Matéria, 2017;22:4.

(16) He W, Benson R. Polymeric Biomaterials. Applied Plastics Engineering Handbook, p.145164.Elsevier, 2017.

(17) Lewin, M. Handbook of Fiber Chemistry. CRC Press, $3^{a}$ ed., p. 101-103, 2006.

(18) Xie D, Leng YX, Jing FJ, Huang N. A brief review of bio-tribology in cardiovascular devices. Biosurf Biotribology, 2015;1:249-262.

(19) Hoshi H, Shinshi T, Takatani S. Third-generation Blood Pumps with Mechanical Noncontact Magnetic Bearings. Artif Organs, 2006;30(5):324-338.

(20) Kosaka R, Nishida M, Maruyama O, Yambe T, Imachi K, Yamane T. Effect of a bearing gap on hemolytic property in a hydrodynamically levitated centrifugal blood pump with a semiopen impeller. Bio-Medical Mat Eng, 2013;23:37-47.

(21) Laang SH, Tung HK, Lai MK, Husnain M, Ashraf M. Design of Linear Tribological Wear Tester. Mechanical System Design, 2016.

(22) Silverstein RM, Webster FX, Kiemle DJ. Spectrometric identification of organic compounds. $7^{\mathrm{a}}$ ed. John Wiley \& Sons, 2005.

(23) Nuffield, E. W. X-Ray Diffraction methods. John Wiley \& Sons, 1966.

(24) Bish DL, Post JE. Quantitative mineralogical analysis using the Rietveld full-pattern fitting method. Am Mineralogist, 1993;78:932-940.

(25) Suryanarayana C, Norton M. X-Ray Diffraction: A Practical Approach. Springer, 2013.

(26) Reitman M, Jaekel D, Siskey R, Kurtz SM. Morphology and Crystalline Architecture of Polyaryletherketones. PEEK Biomaterials Handbook, 2012.

(27) Lai CC, Chen SY, Chen MH, Chen HL, Hsiao HT, Liu LC, Chen CM. Preparation and characterization of heterocyclic polyamide 6 (PA 6) with high transparencies and low hygroscopicities. J Mol Structure, 2019;1175:836-843. 